\title{
Phase space of modified Gauss-Bonnet gravity
}

\author{
Sante Carloni ${ }^{1, a}$, José P. Mimoso ${ }^{2}$ \\ ${ }^{1}$ Centro Multidisciplinar de Astrofísica-CENTRA, Instituto Superior Tecnico-IST, Universidade de Lisboa-UL, Avenida Rovisco Pais 1, \\ 1049-001 Lisbon, Portugal \\ ${ }^{2}$ Departamento de Física, Faculdade de Ciências, Instituto de Astrofísica e Ciências do Espaço, Universidade de Lisboa, Ed. C8, Campo Grande, \\ 1749-016 Lisbon, Portugal
}

Received: 3 January 2017 / Accepted: 27 July 2017 / Published online: 16 August 2017

(C) The Author(s) 2017. This article is an open access publication

\begin{abstract}
We investigate the evolution of non-vacuum Friedmann-Lemaître-Robertson-Walker (FLRW) spacetimes with any spatial curvature in the context of GaussBonnet gravity. The analysis employs a new method which enables us to explore the phase space of any specific theory of this class. We consider several examples, discussing the transition from a decelerating into an acceleration universe within these theories. We also deduce from the dynamical equations some general conditions on the form of the action which guarantee the presence of specific behaviours like the emergence of accelerated expansion. As in $f(R)$ gravity, our analysis shows that there is a set of initial conditions for which these models have a finite time singularity which can be an attractor. The presence of this instability also in the Gauss-Bonnet gravity is to be ascribed to the fourth-order derivative in the field equations, i.e., is the direct consequence of the higher order of the equations.
\end{abstract}

\section{Introduction}

The search for theoretical framework for the now widely accepted increasing expansion rate of the universe, also dubbed "cosmic acceleration", has led the scientific community to consider different possibilities. Some are connected to the presence of additional matter fields with exotic properties (e.g. the inflaton, quintessence, k-essence). Others are related to the geometrical properties of cosmological spacetimes or the special features of the homogeneous and isotropic averaging of an anisotropic and non-homogeneous spacetime. A third class includes modifications (or rather extensions) of the underlying theory of gravitation. This last case implies a purely geometrical description of dark energy and has been thoroughly studied in the past years - from the now "classical" $f(R)[1-4]$ and scalar tensor [5,6] theories to more

\footnotetext{
a e-mail: sante.carloni@gmail.com
}

complex extensions which involve the presence of torsion or of more complicated invariants in the gravitational actions [7-9]. These theories have been thoroughly studied and have revealed interesting features as well as a number of problems connected to different kinds of instabilities. Among those theories which include terms of fourth order in the derivative of the metric, the so-called modified Gauss-Bonnet gravity has been shown to have interesting properties.

The idea of these theories comes from the concept of brane worlds, which are in turn derived from string theories. In five dimensions one considers a Gauss-Bonnet (GB) term which is normally non-minimally coupled with a scalar (modulus or dilaton) field. One can shown that the induced theory on four dimensions is able to generate the de Sitter solution and has other relevant cosmological properties [10-17].

In $[18,19]$ a new class of theories of gravity, dubbed Gauss-Bonnet gravity, was proposed in which the GaussBonnet term appears in four dimensions. As is well known, the Gauss-Bonnet invariant is a total divergence in four dimensions and therefore a linear GB term would be irrelevant in this case. The new class of theories overcomes this issue introducing in the Hilbert-Einstein action a generic non-linear function of the Gauss-Bonnet term. The cosmology of Gauss-Bonnet gravity has been thoroughly studied [20-23]. Some of the members of this class of theories were shown to be able to pass the Solar System tests and to possess cosmological solutions producing cosmic acceleration [24-26]. The same authors also found that linear cosmological perturbations of a spatially flat background contains some instabilities [27]. Although this is clearly a problem, one must remember that the dynamics of higher order theories depends on the value of the spatial curvature in a more complex way that standard (GR). This means that even if $k$ is almost zero, as recently found by the Planck collaboration [28], the dynamical features of these models can be completely different, especially at perturbative level (see Eq. (13) below for a glimpse of this). This fact justifies a further 
analysis of these theories which takes into account spatial curvature.

Gauss-Bonnet gravity, like many other extensions of GR, has a structure that makes it difficult to understand the physics of these models. In this respect, the use of semi quantitative techniques, like the dynamical systems approach (DSA) [29], can help in clarifying details of the dynamics of cosmological models based on these theories and other modifications of GR [30-52] which are not obvious. The use of DSA for this purpose is now fairly standard; however, the analysis is often limited to basic aspects of the method, like for example the choice of variables and the closure do the dynamical system. Recently, a new strategy to alleviate these problems was proposed in the context of $f(R)$-gravity [53]. With respect to the previously proposed approaches, the new method has the advantage to be applicable to any form of the function $f$ and leads to a clearer (albeit not complete) understanding of expanding cosmologies.

In this work, we will propose a similar technique to treat Gauss Bonnet cosmologies in which the action is the sum of the Hilbert-Einstein term and a generic (non linear) function of the Gauss Bonnet invariant. As in the case of $f(R)$ gravity the method will allow one to treat any forms of the function $f$ and will enable us to characterise the nature of the attractors (if any) for the cosmologies Gauss-Bonnet gravity. We will apply this technique to a number of versions of this theory that have been deemed interesting and/or compatible with the present data. The results of this study will be the starting point for a more complete analysis of the dynamics of linear perturbations, which will be pursued elsewhere.

Unless otherwise specified, natural units ( $\hbar=c=k_{B}=$ $G=1$ ) will be used throughout this paper, Latin indices run from 0 to 3 . The symbol $\nabla$ represents the usual covariant derivative and $\partial$ corresponds to partial differentiation. We use the $(+,-,-,-)$ signature and the Riemann tensor is defined by

$R_{b c d}^{a}=W_{b d, c}^{a}-W_{b c, d}^{a}+W^{e}{ }_{b d} W^{a}{ }_{c e}-W^{f}{ }_{b c} W^{a}{ }_{d f}$,

where the $W^{a} b d$ are the Christoffel symbols (i.e. symmetric in the lower indices), defined by

$W_{b d}^{a}=\frac{1}{2} g^{a e}\left(g_{b e, d}+g_{e d, b}-g_{b d, e}\right)$.

The Ricci tensor is obtained by contracting the first and the third indices,

$R_{a b}=g^{c d} R_{a c b d}$.

Finally the Hilbert-Einstein action in the presence of matter is given by

$S=\int d x^{4} \sqrt{-g}\left[\frac{1}{2 \kappa} R+L_{m}\right]$ where $\kappa=8 \pi$ and has the dimension of the inverse of a length square.

\section{Basic equations}

The action for modified Gauss-Bonnet gravity reads

$S=\int \mathrm{d}^{4} x \sqrt{-g}\left[\frac{R}{2 \kappa}+f(\mathcal{G})\right]+S_{\mathrm{M}}\left(g^{\mu \nu}, \psi\right)$,

where $S_{M}\left(g^{\mu \nu}, \psi\right)$ is the matter action and $\psi$ collectively denotes the matter fields. The Gauss-Bonnet invariant is defined as

$\mathcal{G} \equiv R^{2}-4 R_{\mu \nu} R^{\mu \nu}+R_{\mu \nu \alpha \beta} R^{\mu \nu \alpha \beta}$

Varying the action with respect to the metric yields the field equations:

$$
\begin{aligned}
& G_{\mu \nu}+\left(\mathcal{G} f^{\prime}-f\right) g_{\mu \nu} \\
& \quad+8\left[R_{\mu \rho \nu \sigma}+R_{\rho \nu} g_{\sigma \mu}-R_{\rho \sigma} g_{\nu \mu}-R_{\mu \nu} g_{\sigma \rho}+R_{\mu \sigma} g_{\nu \rho}\right. \\
& \left.\quad+\frac{R}{2}\left(g_{\mu \nu} g_{\sigma \rho}-g_{\mu \sigma} g_{\nu \rho}\right)\right] \nabla^{\rho} \nabla^{\sigma} f^{\prime}=\kappa T_{\mu \nu},
\end{aligned}
$$

where the prime represents the derivative with respect to $\mathcal{G}$ and $G_{\mu \nu}=R_{\mu \nu}-(1 / 2) R g_{\mu \nu}$ is the Einstein tensor.

The matter energy-momentum tensor is defined as usual as

$T_{\mu \nu}=-\frac{2}{\sqrt{-g}} \frac{\delta\left(\sqrt{-g} L_{m}\right)}{\delta\left(g^{\mu \nu}\right)}$.

Our treatment will consider the Friedmann-LemaîtreRobertson-Walker (FLRW) metric:

$\mathrm{d} s^{2}=-\mathrm{d} t^{2}+a^{2}(t)\left[\frac{\mathrm{d} r^{2}}{1-k r^{2}}+r^{2}\left(\mathrm{~d} \theta^{2}+\sin ^{2} \theta \mathrm{d} \phi^{2}\right)\right]$,

where $a$ is the scale factor and $k$ the spatial curvature. We also assume that the cosmic fluid is a perfect fluid with equation of state $p=w \mu$ with $0 \leq w \leq 1$, where $\mu$ and $p$, respectively are the energy density and pressure measured by the co-moving observer, and where we assume $w$ to be constant. In this metric, the Gauss-Bonnet invariant is

$\mathcal{G}=24\left(H^{2}+\frac{k}{a^{2}}\right)\left(\dot{H}+H^{2}\right)$

where $H=\frac{\dot{a}}{a}$ is the Hubble factor. This expression is particularly important because it connects the sign of $\mathcal{G}$ to the sign of the deceleration factor. For $k \geq 0$, any cosmology which transits between acceleration and deceleration will have to 
change the sign of $\mathcal{G}$. If $k<0$, this is not necessarily true. In this respect Gauss-Bonnet cosmology depends in an even more crucial way on the spatial curvature than GR.

\section{The dynamical systems approach}

First, we introduce a constant $\chi_{0}$ such that the products $R \chi_{0}$ and $\mathcal{G} \chi_{0}^{2}$ are dimensionless and we redefine all the constants appearing in the action (5) to obtain

$$
S=\int\left\{\chi_{0} R+f\left(\mathcal{G} \chi_{0}^{2}\right)+L_{m}\left(g_{\mu \nu}, \Phi\right)\right\} \sqrt{-g} \mathrm{~d}^{4} x,
$$

where now all the constants (with the exception of $\chi_{0}$ ) in the action are assumed dimensionless.

Now defining the parameters

$\mathfrak{q}=\frac{\dot{H}}{H^{2}}, \quad \mathfrak{j}=\frac{\ddot{H}}{H^{3}}-\frac{\dot{H}^{2}}{H^{4}}, \quad \mathfrak{s}=\frac{\dddot{H}}{H^{4}}+3 \frac{\dot{H}^{3}}{H^{6}}-4 \frac{\dot{H} \ddot{H}}{H^{5}}$,

the cosmological equations can be written as

$$
\begin{aligned}
& 3 \chi_{0}\left(H^{2}+\frac{k}{a^{2}}\right)-\mu+f-24 H^{2}(\mathfrak{q}+1)\left(H^{2}+\frac{k}{a^{2}}\right) f^{\prime} \\
& +\left\{3^{2} 2^{6} H^{4}\left(\mathfrak{j}+\mathfrak{q}^{2}-2\right) \frac{k^{2}}{a^{4}}\right. \\
& +3^{2} 2^{7} H^{6}(5 \mathfrak{j}+2 \mathfrak{q}(3+1)-9) \\
& \left.\quad \times \frac{k}{a^{2}}+2^{6} 3^{4} H^{8}[\mathfrak{j}+\mathfrak{q}(3 \mathfrak{q}+4)]\right\} f^{\prime \prime}=0, \\
& \chi_{0} H^{2}(\mathfrak{q}+1)+\frac{1}{3} \mu(3 w+1)+\frac{f}{3} \\
& -24 H^{2}(\mathfrak{q}+1)\left(\frac{k}{a^{2}}+H^{2}\right) f^{\prime} \\
& +\left\{2^{5} 3^{2} H^{4}\left(\frac{k}{a^{2}}+H^{2}\right)\left(\frac{k}{a^{2}}+9 H^{2}\right) \mathfrak{s}\right. \\
& +2^{5} 3^{2} H^{8}\left[\mathfrak{j}(92 \mathfrak{q}+29)+\mathfrak{q}^{2}(87 \mathfrak{q}+131)-28 \mathfrak{q}\right] \\
& +2^{5} 3^{2} H^{4}\left[\mathfrak{j}(4 \mathfrak{q}-3)+(\mathfrak{q}-3) \mathfrak{q}^{2}+6(\mathfrak{q}-1)\right] \frac{k^{2}}{a^{4}} \\
& \left.+2^{6} 3^{2} H^{6}[\mathfrak{j}(24 \mathfrak{q}-11)+\mathfrak{q}(2 \mathfrak{q}(5 \mathfrak{q}-3)-31)+25] \frac{k}{a^{2}}\right\} f^{\prime \prime} \\
& +\left\{2^{8} 3^{3} H^{6}\left(\mathfrak{j}+\mathfrak{q}^{2}-2\right) \frac{k^{3}}{a^{6}}+2^{8} 3^{3} H^{8}\left(\mathfrak{j}+\mathfrak{q}^{2}-2\right)\right. \\
& \quad \times[11 \mathfrak{j}+\mathfrak{q}(15 \mathfrak{q}+8)-18] \frac{k^{2}}{a^{4}} \\
& +2^{8} 3^{3} H^{10}[\mathfrak{j}+\mathfrak{q}(3 \mathfrak{q}+4)][19 \mathfrak{j}+\mathfrak{q}(21 \mathfrak{q}+4)-36] \\
& \left.\times \frac{k}{a^{2}}+2^{8} 3^{5} H^{12}[\mathfrak{j}+\mathfrak{q}(3 \mathfrak{q}+4)]^{2}\right\} f^{\prime \prime \prime}=0 .
\end{aligned}
$$

$$
\begin{aligned}
\mathbb{G} & =\frac{\mathcal{G}}{3 H^{4}}, \quad \mathbb{K}=\frac{k}{a^{2} H^{2}}, \quad \Omega=\frac{\mu}{3 H^{2} \chi_{0}}, \\
\mathbb{J} & =\mathfrak{j} \quad \mathbb{Q}=\mathfrak{q}, \quad \mathbb{A}=\chi_{0} H^{2} .
\end{aligned}
$$

We also define the logarithmic (dimensionless) "time variable" $N=\ln a$. Note that in choosing this time variable we are assuming that we represent the phase space for $H>0$, i.e., we are considering only expanding cosmologies.

It is important to stress that, since $\mathbb{G}$ has the same sign of $\mathcal{G}$, a sufficient condition for the transition between deceleration and acceleration can only be obtained if $\mathbb{G}=0$ is not an invariant submanifold of the phase space.

The phase space associated to Eq. (13) is then described by the autonomous system

$$
\begin{aligned}
& \frac{\mathrm{d} \mathbb{G}}{\mathrm{d} N}=\frac{\mathbb{G}}{2}\left(8-\frac{\mathbb{G}}{\mathbb{K}+1}\right)-\frac{8[\mathbb{K}+\mathbf{X}-\mathbb{G} \mathbf{Y}-\Omega+1]}{(\mathbb{K}+9) \mathbf{Z}}, \\
& \frac{\mathrm{d} \Omega}{\mathrm{d} N}=\Omega\left[\frac{\mathbb{G}}{4(\mathbb{K}+1)}+(3 w+1)\right], \\
& \frac{\mathrm{d} \mathbb{K}}{\mathrm{d} N}=-\frac{\mathbb{K} \mathbb{G}}{4 \mathbb{K}+4}, \\
& \frac{\mathrm{d} \mathbb{A}}{\mathrm{d} N}=\frac{\mathbb{A}}{4}\left(\frac{\mathbb{G}}{\mathbb{K}+1}-8\right),
\end{aligned}
$$

together with the two constraints

$$
\begin{aligned}
& (9+\mathbb{K})\left[\mathbb{J}+\mathbb{K}\left(\mathbb{J}+\mathbb{Q}^{2}-2\right) \mathbf{Z}+\mathbb{Q}(3 \mathbb{Q}+4)\right] \\
& \quad+\mathbb{K}+\mathbf{X}-\mathbb{G} \mathbf{Y}-\Omega+1=0 \\
& \mathbb{G}=8(1+\mathbb{K})(1+\mathbb{Q}) .
\end{aligned}
$$

In all the equations above we have defined

$$
\begin{aligned}
& \mathbf{X}=\frac{f}{3 \chi_{0} H^{2}}, \\
& \mathbf{Y}=\frac{f^{\prime} H^{2}}{\chi_{0}}, \\
& \mathbf{Z}=\frac{32^{6} H^{6} f^{\prime \prime}}{\chi_{0}} .
\end{aligned}
$$

Note that choosing the variables above, the system is singular for $\mathbb{K}+1=0$. Such a singularity originates with our choice of coordinates: selecting for example the variable $\mathbb{Q}$ instead of $\mathbb{G}$ it can be eliminated to obtain the system 


$$
\begin{aligned}
\frac{\mathrm{d} \mathbb{Q}}{\mathrm{d} N}= & -\mathbb{Q}^{2}-\frac{\left(\mathbb{Q}^{2}-2\right) \mathbb{K}+\mathbb{Q}(3 \mathbb{Q}+4)}{(\mathbb{K}+1)} \\
& -\frac{\mathbb{K}-\Omega+1+\mathbf{X}-8(\mathbb{K}+1)(\mathbb{Q}+1) \mathbf{Y}}{(\mathbb{K}+1)(\mathbb{K}+9) \mathbf{Z}}, \\
\frac{\mathrm{d} \Omega}{\mathrm{d} N}= & -\Omega[2 \mathbb{Q}+3(w+1)], \\
\frac{\mathrm{d} \mathbb{K}}{\mathrm{d} N}= & -2 \mathbb{K}(1+\mathbb{Q}), \\
\frac{\mathrm{d} \mathbb{A}}{\mathrm{d} N}= & \mathbb{A} \mathbb{Q},
\end{aligned}
$$

where now $\mathbf{X}, \mathbf{Y}, \mathbf{Z}$ are functions of $\mathbb{Q}, \mathbb{K}$. The two systems are equivalent when one is away from $\mathbb{K}=-1$. Since for the examples we consider there is no special point for $\mathbb{K}=-1$ and, moreover, there is no appreciable difference in the structure of the fixed points, we will perform the analysis using Eq. (16). However, one must stress that $\mathbb{K}=-1$ is nevertheless of interest because, since both $\mathbb{G}$ and its derivatives become zero, this case represents a state in which the theory effectively becomes of order two. Therefore the system (22) can be useful to explore this property of Gauss-Bonnet gravity.

The solutions associated to the fixed points can be derived using the modified Raychaudhury equation [the second of the Eq. (13)] in the variables (12). At a fixed point we have

$$
\begin{aligned}
\frac{1}{H} \frac{\mathrm{d}^{3} H}{\mathrm{~d} N^{3}}= & \mathfrak{s}_{*}, \\
\mathfrak{s}_{*}= & -\frac{1}{\left(K_{*}+1\right)\left(K_{*}+9\right) \mathbf{Z}_{*}}\left\{2\left(K_{*}+9\right) \mathbf{T}_{*}\right. \\
& \times\left[\mathbb{J}_{*}+K_{*}\left(\mathbb{J}_{*}+\mathbb{Q}_{*}^{2}-2\right)+\mathbb{Q}_{*}\left(3 \mathbb{Q}_{*}+4\right)\right]^{2} \\
& +2 \mathbb{Q}_{*}+(1+3 w) \Omega_{*}+2 \mathbf{X}_{*}-2 \mathbb{G}_{*} \mathbf{Y}_{*}+2 \\
& +29 \mathbf{Z}_{*} \mathbb{J}_{*}+2 \mathbf{Z}_{*} \mathbb{Q}_{*}\left[46 \mathbb{J}_{*}+2 K_{*} \mathbb{J}_{*}\left(K_{*}+12\right)\right. \\
& \left.-K_{*}\left(3 K_{*}+31\right)\right]+K_{*} \mathbf{Z}_{*}\left[50-22 \mathbb{J}_{*}-3 \mathbb{J}_{*} K_{*}\right. \\
& \left.+6 K_{*}\right]+\mathbf{Z}_{*}\left[K_{*}\left(K_{*}+20\right)+87\right] \mathbb{Q}_{*}^{3} \\
& \left.+\mathbf{Z}_{*}\left[131-3 K_{*}\left(K_{*}+4\right)\right] \mathbb{Q}_{*}^{2}-28 \mathbf{Z}_{*} \mathbb{Q}_{*}\right\}
\end{aligned}
$$

where

$\mathbf{T}=\frac{2^{8} 3^{2} H^{10} f^{\prime \prime \prime}}{\chi_{0}}$

and an asterisk represents the value of a quantity at a fixed point. The general solution for the above equation is

$H= \begin{cases}H_{0}+H_{1} N+H_{2} N^{2} & \mathfrak{s}^{*}=0, \\ H_{0} e^{-p N}+e^{\frac{p N}{2}}\left(H_{1} \cos \frac{p N \sqrt{3}}{2}\right. & \\ \left.+H_{2} \sin \frac{p N \sqrt{3}}{2}\right) & \mathfrak{s}^{*} \neq 0,\end{cases}$ where $p=-\sqrt[3]{\mathfrak{s}^{*}}$ and the $H_{i}$ are integration constants. Using the definition of $N$ the above equations translate in equations for the scale factor

$$
\frac{\dot{a}}{a}= \begin{cases}H_{0}+H_{1} \ln a+H_{2}(\ln a)^{2} & \mathfrak{s}^{*}=0 \\ H_{0} a^{-p}+a^{p / 2}\left[H_{1} \cos \left(\frac{p \sqrt{3}}{2} \ln a\right)\right. & \\ \left.+H_{2} \sin \left(\frac{p \sqrt{3}}{2} \ln a\right)\right] & \mathfrak{s}^{*} \neq 0\end{cases}
$$

In the first case the equation can be solved exactly to give

$$
\begin{aligned}
a(t)= & a_{0} \exp \left\{\frac{\sqrt{4 H_{2} H_{0}-H_{1}^{2}}}{2 H_{2}}\right. \\
& \left.\times \tan \left[\frac{1}{2}\left(t-t_{0}\right) \sqrt{4 H_{2} H_{0}-H_{1}^{2}}\right]-\frac{H_{1}}{2 H_{2}}\right\},
\end{aligned}
$$

which was already found in the case of $f(R)$-gravity [53].

A major problem when these solutions are found is associated with the determination of the integration constants. Such constants are important as they concur to determine the nature of the solution. For example, the solution given by (27) will have a finite time singularity if $4 \mathrm{H}_{2} \mathrm{H}_{0}-\mathrm{H}_{1}^{2}>0$. One way in which the value of these constants can be inferred is to consider the value of the initial condition of the orbits that approach this point.

\section{Examples}

We will now apply the machinery presented above to a number of physically relevant theories. We will then compare our results with those found in the literature.

\section{$4.1 \mathcal{G}^{n}$-gravity}

As a first example, let us consider the model

$f=\alpha \chi_{0}^{2 n} \mathcal{G}^{n}$

we have

$$
\begin{aligned}
& \mathbf{X}=3^{n-1} \alpha \mathbb{A}^{2 n-1} \mathbb{G}^{n}, \\
& \mathbf{Y}=3^{n-1} n \alpha \mathbb{A}^{2 n-1} \mathbb{G}^{n-1}, \\
& \mathbf{Z}=2^{6} 3^{n-1}(n-1) n \alpha \mathbb{A}^{2 n-1} \mathbb{G}^{n-2}, \\
& \mathbf{T}=2^{8} 3^{n-1}(n-2)(n-1) n \alpha \mathbb{A}^{2 n-1} \mathbb{G}^{n-3},
\end{aligned}
$$

where the constraint (18) holds. The dynamical equations read 


$$
\begin{aligned}
& \frac{\mathrm{d} \mathbb{G}}{\mathrm{d} N}= \mathbb{G}\left[4-\mathbb{G}\left(\frac{1}{\mathbb{K}+1}+\frac{1}{4 n(\mathbb{K}+9)}\right)\right. \\
&\left.+\frac{2^{2-n} 3^{1-n} \mathbb{A}^{1-2 n}(\mathbb{K}-\Omega+1) \mathbb{G}^{1-n}}{n(1-n) \alpha(\mathbb{K}+9)}\right], \\
& \frac{\mathrm{d} \Omega}{\mathrm{d} N}=\Omega\left[\frac{\mathbb{G}}{4(\mathbb{K}+1)}+(3 w+1)\right], \\
& \frac{\mathrm{d} \mathbb{K}}{\mathrm{d} N}=-\frac{\mathbb{K} \mathbb{G}}{4(\mathbb{K}+1)}, \\
& \frac{\mathrm{d} \mathbb{A}}{\mathrm{d} N}=\frac{\mathbb{A}}{4}\left(\frac{\mathbb{G}}{\mathbb{K}+1}-8\right) .
\end{aligned}
$$

The system admits four invariant submanifolds $(\mathbb{K}=0, \Omega=$ $0, \mathbb{A}=0, \mathbb{G}=0)$. These results imply that no global attractor exists in general.

The fixed subspaces for this system are indicated in Table 1 . We have a line of fixed points $\mathcal{L}$ for $\mathbb{K}=\mathbb{K}_{0}, \Omega=$ $0, \mathbb{A}=0, \mathbb{G}=0$ for $2+3 n<0$. Other fixed points exist only at specific intervals of $n$. For example, the point $\mathcal{A}$ is present for $2-3 n>0$, whereas $\mathcal{B}$ is present only for $(n-1) \alpha>0$.

The points on the line $\mathcal{L}$ are associated with the solution given by the equation

$$
\begin{gathered}
\frac{\dot{a}}{a}=\frac{H_{1}}{a}+a^{1 / 2}\left[H_{2} \sin \left(\frac{1}{2} \sqrt{3} \log a\right)\right. \\
\left.+H_{3} \cos \left(\frac{1}{2} \sqrt{3} \log a\right)\right] .
\end{gathered}
$$

For point $\mathcal{A}$ we have

$$
\begin{aligned}
\frac{\dot{a}}{a}= & H_{1} a^{\frac{1-36 n}{\sqrt[3]{2^{9} 3^{3} n^{2}+1}}}+a^{1 / 2}\left[H_{2} \sin \left(\frac{(1-36 n) \sqrt{3}}{\sqrt[3]{2^{10} 3^{3} n^{2}+1}} \log a\right)\right. \\
& \left.+H_{3} \cos \left(\frac{(1-36 n) \sqrt{3}}{\sqrt[3]{2^{10} 3^{3} n^{2}+1}} \log a\right)\right]
\end{aligned}
$$

whereas $\mathcal{B}$ corresponds to solution (27). A numerical integration of Eqs. (34) and (35) is given in Figs. 1 and 2, and in Fig. 3 we plot the solution of Eq. (27).

The stability of all the fixed points can be deduced using the standard Hartmann-Grobmann theorem [54,55] and it is indicated in Table 1 . The points on $\mathcal{L}$ are all unstable and $\mathcal{A}$ and $\mathcal{B}$ can be attractors or saddles depending on the values of $n$ and $\alpha$.

Because of the presence of the invariant submanifold there is no orbit that can represent a transition between decelerated and accelerated cosmologies. In this respect this theory is not useful to model dark energy and could only be used as patch model for eternal inflation. Even in this case, however, these models can have the same issues of $f(R)$ gravity, i.e. the onset of a finite time singularity. Our conclusions are consistent with the results in $[24,26]$.

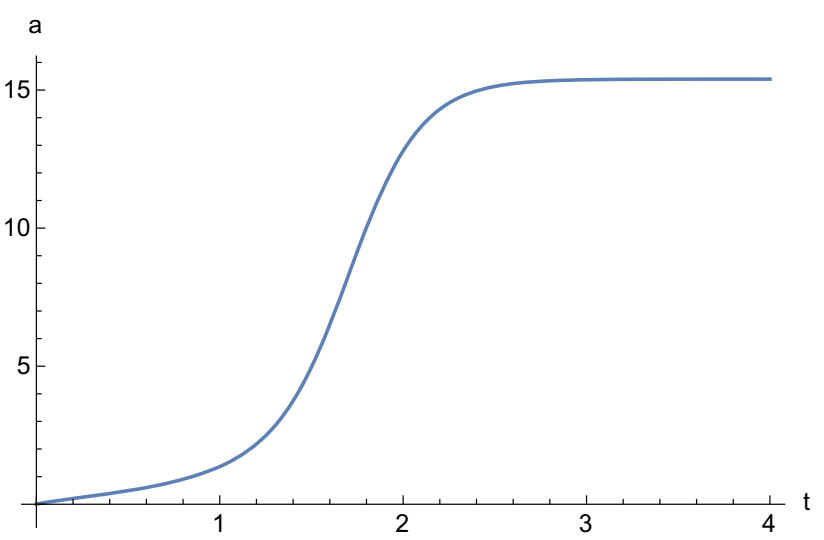

Fig. 1 Numerical solution of Eq. (34). The constants $H_{i}$ have all been chosen to be one and the initial condition is $a(0)=0.01$

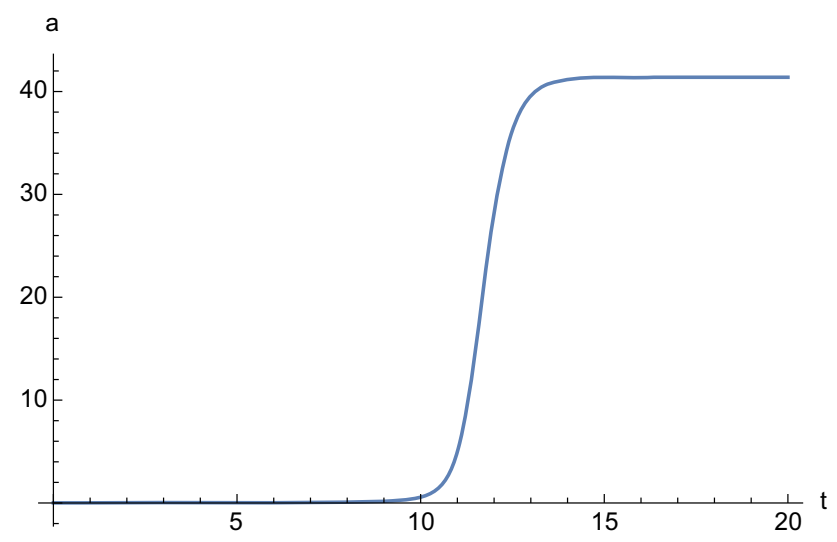

Fig. 2 Numerical solution of Eq. (35). The constants $H_{i}$ have all been chosen to be one and the initial condition is $a(0)=0.01$

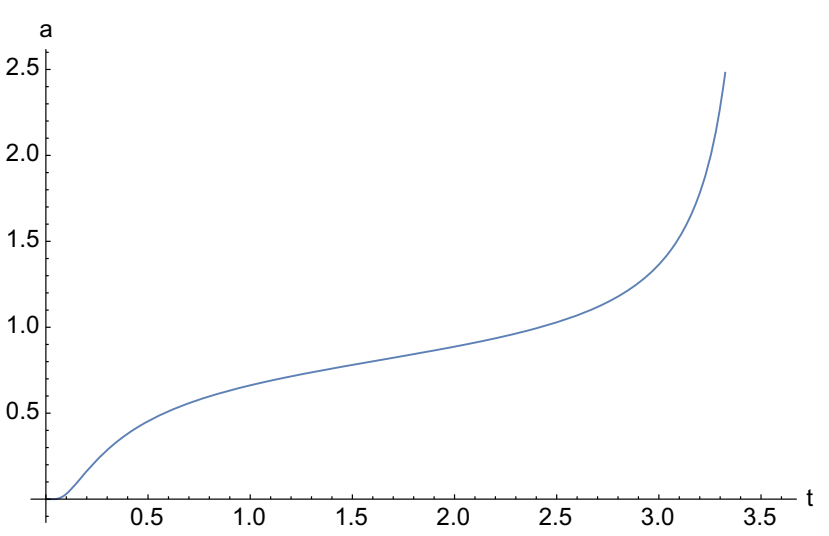

Fig. 3 Numerical solution of Eq. (27). The constants $H_{i}$ have all been chosen in such a way that $4 H_{0} H_{2}-H_{1}^{2}>0$ and the initial condition is $a(0)=0.01$. The solution presents a finite time singularity. If the above condition is violated the solution approaches a static universe as in the case of (34) and (35)

\subsection{The De Felice-Tsujikawa models}

In Ref. [25] some particular forms of $f(G)$ were proposed which, based on exact and perturbative arguments, were con- 
Table 1 Fixed subspaces of $f(\mathcal{G})=\alpha \mathcal{G}^{n}$ and their associated solutions. Here $a_{0}=H_{1}+H_{2}+H_{3}$, A stays for attractor, $\mathrm{F}_{A}$ for attractive focus, $\mathrm{S}$ for saddle

\begin{tabular}{lllll}
\hline & Coordinates $\{\mathbb{G}, \mathbb{K}, \Omega, \mathbb{A}\}$ & Scale factor & Existence & Stability \\
\hline $\mathcal{L}$ & $\left\{0, \mathbb{K}_{0}, 0,0\right\}$ & Solution of (34) & $2-3 n>0$ & Unstable \\
$\mathcal{A}$ & $\left\{\frac{2^{5} 3^{2}}{36 n-1}, 0,0,0\right\}$ & Solution of (35) & $2-3 n>0$ & $\mathrm{~A} n<0$ \\
& & & & $\mathrm{~S} 0<n<2 / 3$ \\
$\mathcal{B} \quad\left\{8,0,0,\left[3^{n-1} 8^{n}(n-1) \alpha\right]^{\frac{1}{1-2 n}}\right\}$ & Equation $(27)$ & $(n-1) \alpha>0$ & $\mathrm{~A} \frac{72}{1369} \leq n<\frac{1}{2}$ \\
& & & & $\mathrm{~F}_{A} 0<n<\frac{72}{1369}$ \\
& & & S otherwise \\
\hline
\end{tabular}

sidered to be cosmologically viable. In our formulation such models can be written as

$f(\mathcal{G})=\alpha \chi_{0}^{2} \mathcal{G} \arctan \left(\chi_{0}^{2} \mathcal{G}\right)-\beta \chi_{0}$,

$f(\mathcal{G})=\alpha \chi_{0}^{2} \mathcal{G} \arctan \left(\chi_{0}^{2} \mathcal{G}\right)-\beta \chi_{0}-\gamma \ln \left(1+\chi_{0}^{2} \mathcal{G}\right)$,

$f(\mathcal{G})=\alpha \ln \left[\cosh \left(\chi_{0}^{2} \mathcal{G}\right)\right]-\beta \chi_{0}$,

where $\beta$, differently form $\alpha$ and $\gamma$, is a dimensional constant. All these constant are considered positive. The presence of $\beta$ requires the introduction of a new variable $\mathbb{B}=\beta / H^{2}$ whose dynamic equation is

$\frac{\mathrm{d} \mathbb{B}}{\mathrm{d} N}=-\frac{3 \mathbb{B}(\mathbb{G}-8 \mathbb{K}-8)}{4(\mathbb{K}+1)}$.

By including this additional equation in the system (16), we are ready to explore these models.

\subsubsection{Moldel 1: $f(\mathcal{G})=\alpha \chi_{0}^{2} \mathcal{G} \arctan \left(\chi_{0}^{2} \mathcal{G}\right)-\beta \chi_{0}$}

For the first form of $f$ above we have

$\mathbf{X}=\alpha \mathbb{A} \mathbb{G} \arctan \left(3 \mathbb{A}^{2} \mathbb{G}\right)-\mathbb{B}$,

$\mathbf{Y}=\alpha \mathbb{A}\left[\arctan \left(3 \mathbb{A}^{2} \mathbb{G}\right)+\frac{3 \mathbb{A}^{2} \mathbb{G}}{9 \mathbb{A}^{4} \mathbb{G}^{2}+1}\right]$,

$\mathbf{Z}=\frac{\alpha 32^{7} \mathbb{A}^{3}}{\left(9 \mathbb{A}^{4} \mathbb{G}^{2}+1\right)^{2}}$,

$\mathbf{T}=-\frac{\alpha 2^{11} 3^{3} \mathbb{A}^{7} \mathbb{G}}{\left(9 \mathbb{A}^{4} \mathbb{G}^{2}+1\right)^{3}}$,

and the dynamical equations read

$$
\begin{aligned}
\frac{\mathrm{d} \mathbb{G}}{\mathrm{d} N}= & \frac{1}{2} \mathbb{G}\left(8-\frac{\mathbb{G}}{\mathbb{K}+1}\right)+\frac{9 \mathbb{A}^{4} \mathbb{G}^{2}+1}{48 \alpha \mathbb{A}^{3}(K+9)} \\
& \times\left\{1+K-\Omega-\mathbb{B}-3 \mathbb{A}^{3} \mathbb{G}^{2}[\alpha+3 \mathbb{A}(\mathbb{B}+\Omega)]\right. \\
& \left.+9 \mathbb{A}^{4} \mathbb{G}^{2}(K+1)\right\}, \\
\frac{\mathrm{d} \Omega}{\mathrm{d} N}= & \Omega\left[\frac{\mathbb{G}}{4(\mathbb{K}+1)}+(3 w+1)\right],
\end{aligned}
$$

$\frac{\mathrm{d} \mathbb{K}}{\mathrm{d} N}=-\frac{\mathbb{K} \mathbb{G}}{4(\mathbb{K}+1)}$,

$\frac{\mathrm{d} \mathbb{A}}{\mathrm{d} N}=\frac{\mathbb{A}}{4}\left(\frac{\mathbb{G}}{\mathbb{K}+1}-8\right)$,

$\frac{\mathrm{d} \mathbb{B}}{\mathrm{d} N}=-\frac{3 \mathbb{B}(\mathbb{G}-8 \mathbb{K}-8)}{4(\mathbb{K}+1)}$.

The system admits three invariant submanifolds $(\mathbb{K}=$ $0, \Omega=0, \mathbb{A}=0$ ). The last one, depending on the values of the parameters, can be singular. These results imply that no global attractor exists in general, but they allow the possibility of a transition between decelerated and accelerated cosmologies.

The system (42) presents only a two dimensional fixed point subspace with coordinates

$$
\begin{aligned}
\mathcal{S} & =\left\{\mathbb{G}_{*}, \mathbb{K}_{*}, \Omega_{*}, \mathbb{A}_{*}, \mathbb{B}_{*}\right\} \\
& =\left\{8,0,0, \mathbb{A}_{*}, 1-\frac{192 \alpha \mathbb{A}_{*}^{3}}{576 \mathbb{A}_{*}^{4}+1}\right\},
\end{aligned}
$$

which corresponds to the solution (27). Note that, by definition, $\mathbb{B}>0$ and this implies that this subspace can exist only for $\left(\alpha<\frac{576 A_{*}^{4}+1}{192 A_{*}^{3}}\right)$.

The stability of the fixed points on the surface $\mathcal{S}$ can be obtained using the Hartmann-Grobmann theorem [54,55]. A plot of the real part of the eigenvalues is given in Fig. 4. It is evident that the points on $\mathcal{S}$ can be saddles or attractors

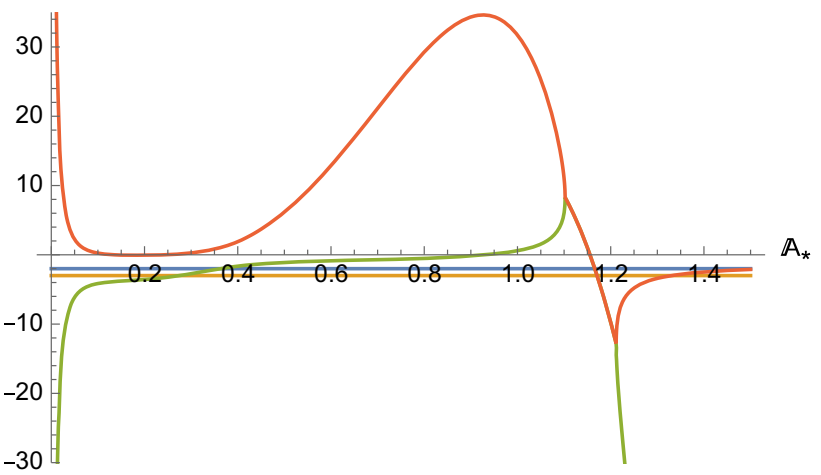

Fig. 4 Plot of the real part of the eigenvalues of the fixed points of the line $\mathcal{L}$ for $f(\mathcal{G})=\alpha \chi_{0}^{2} \mathcal{G} \arctan \left(\chi_{0}^{2} \mathcal{G}\right)-\beta \chi_{0}^{2}$. Here $\alpha$ has been chosen to be 3 and $w=0$ 
depending on the values of $\alpha$. An easier way to visualise the phase space dynamics is to define the variable

$$
\mathbb{Y}=\mathbb{B}-\frac{3 A\left(576 \mathbb{A}^{4}-192 \alpha \mathbb{A}^{3}+1\right)}{\mathbb{A}^{2}\left(576 \mathbb{A}^{4}+1\right)}
$$

and analyse the corresponding dynamical system:

$$
\begin{aligned}
\frac{\mathrm{d} \mathbb{G}}{\mathrm{d} N}= & \frac{1}{2} \mathbb{G}\left(8-\frac{\mathbb{G}}{\mathbb{K}+1}\right)+\frac{9 \mathbb{A}^{4} \mathbb{G}^{2}+1}{48 \alpha \mathbb{A}^{3}(K+9)} \\
& \times\left\{1+K-\Omega-3 \mathbb{A}^{3} \mathbb{G}^{2}(\alpha+3 \mathbb{A} \Omega)\right. \\
& \left.+9 \mathbb{A}^{4} \mathbb{G}^{2}(K+1)\right\} \\
& +\frac{\left(9 A^{4} \mathbb{G}^{2}+1\right)^{2}}{A(K+9)}\left(\frac{A \mathbb{Y}+3}{48 \alpha A^{3}}-\frac{12}{576 A^{4}+1}\right), \\
\frac{\mathrm{d} \Omega}{\mathrm{d} N}= & \Omega\left[\frac{\mathbb{G}}{4(\mathbb{K}+1)}+(3 w+1)\right], \\
\frac{\mathrm{d} \mathbb{K}}{\mathrm{d} N}= & -\frac{\mathbb{K} \mathbb{G}}{4(\mathbb{K}+1)}, \\
\frac{\mathrm{d} \mathbb{A}}{\mathrm{d} N}= & \frac{\mathbb{A}}{4}\left(\frac{\mathbb{G}}{\mathbb{K}+1}-8\right), \\
\frac{\mathrm{d} \mathbb{Y}}{\mathrm{d} N}= & \frac{3(-\mathbb{G}+8 K+8)}{4 \mathbb{A}\left(576 \mathbb{A}^{4}+1\right)^{2}(K+1)}\left[\left(576 \mathbb{A}^{4}+1\right)^{2}\right. \\
& \left.\times(\mathbb{Y}+2)-192 \alpha \mathbb{A}^{3}\left(576 \mathbb{A}^{4}+5\right)\right],
\end{aligned}
$$

which presents only one line of fixed points $\left(\left\{8,0,0, \mathbb{A}_{*}, 0\right\}\right)$ rather than a surface and shows clearly that there is no heteroclinic orbit that connects the unstable points to the stable points on the line. This implies that there cannot be an orbit in which an unstable phase characterised by (27) is followed by a stable phase characterised by the same solution.

This implies that the theory has an accelerated expansion attractor, but in order to conclude that a transition deceleration/acceleration is possible one should look at the numerical evolution of the orbits. This result is consistent with what was found in [26], in which one of such transitions has been observed by numerical integration.

There is no general way to deduce the size of the set of initial conditions that leads to cosmic acceleration, however, by inspection, we can deduce that the smaller the value of the parameter $\alpha$ the larger the initial conditions that lead to the cosmic acceleration transition.

In this model the presence of the cosmological constant does not seem to change too much the dynamics. When $\mathbb{B}=0$ the fixed subspace reduces to a one dimensional space of fixed points associated to the solution (27). Their character can be once again either that of a saddle or of an attractor, depending on the value of $\alpha$. Again the bigger the parameter $\alpha$ the lower the number of stable points in the one dimensional space. The only concrete difference between this model and the more general one is the fact that the equations to solve are easier in the presence of the cosmological constant.

$$
\begin{aligned}
& \text { 4.2.2 Model 2: } f(\mathcal{G})=\alpha \chi_{0}^{2} \mathcal{G} \arctan \left(\chi_{0}^{2} \mathcal{G}\right)-\gamma \ln \\
& \left(1+\chi_{0}^{2} \mathcal{G}\right)-\beta \chi_{0}
\end{aligned}
$$

For the form of $f$ above we have

$$
\begin{aligned}
\mathbf{X}= & -\frac{\gamma \log \left(9 \mathbb{A}^{4} \mathbb{G}^{2}+1\right)}{3 \mathbb{A}} \\
& +\alpha \mathbb{A} \mathbb{G} \tan ^{-1}\left(3 \mathbb{A}^{2} \mathbb{G}\right)-\mathbb{B} \\
\mathbf{Y}= & \alpha \mathbb{A} \tan ^{-1}\left(3 \mathbb{A}^{2} \mathbb{G}\right)+\frac{3 \mathbb{A}^{3} \mathbb{G}(\alpha-2 \gamma)}{9 \mathbb{A}^{4} \mathbb{G}^{2}+1} \\
\mathbf{Z}= & \frac{384 \mathbb{A}^{3}\left(\alpha+\gamma\left(9 \mathbb{A}^{4} \mathbb{G}^{2}-1\right)\right)}{\left(9 \mathbb{A}^{4} \mathbb{G}^{2}+1\right)^{2}} \\
\mathbf{T}= & -\frac{27648 \mathbb{A}^{7} \mathbb{G}\left(2 \alpha+9 \mathbb{A}^{4} \gamma \mathbb{G}^{2}-3 \gamma\right)}{\left(9 \mathbb{A}^{4} \mathbb{G}^{2}+1\right)^{3}}
\end{aligned}
$$

and the dynamical equations read

$$
\begin{aligned}
\frac{\mathrm{d} \mathbb{G}}{\mathrm{d} N}= & \frac{1}{2} \mathbb{G}\left(8-\frac{\mathbb{G}}{\mathbb{K}+1}\right)+\frac{9 \mathbb{A}^{4} \mathbb{G}^{2}+1}{48 \alpha \mathbb{A}^{3}(K+9)} \\
& \times\left\{1+K-\Omega-\mathbb{B}-3 \mathbb{A}^{3} \mathbb{G}^{2}[\alpha+3 \mathbb{A}(\mathbb{B}+\Omega)]\right. \\
& \left.+9 \mathbb{A}^{4} \mathbb{G}^{2}(K+1)\right\} \\
& +\frac{\gamma\left(9 A^{4} \mathbb{G}^{2}+1\right)^{2}}{144 A^{4}(K+9)\left(\alpha-\gamma+9 A^{4} \gamma \mathbb{G}^{2}\right)} \\
& \times \ln \left(9 A^{4} \mathbb{G}^{2}+1\right) \\
\frac{\mathrm{d} \Omega}{\mathrm{d} N}= & \Omega\left[\frac{\mathbb{G}}{4(\mathbb{K}+1)}+(3 w+1)\right] \\
\frac{\mathrm{d} \mathbb{K}}{\mathrm{d} N}= & -\frac{\mathbb{K} \mathbb{G}}{4(\mathbb{K}+1)}, \\
\frac{\mathrm{d} \mathbb{B}}{\mathrm{d} N}= & -\frac{3 \mathbb{B}(\mathbb{G}-8 \mathbb{K}-8)}{4(\mathbb{K}+1)} .
\end{aligned}
$$

The system above differs for the previous one only by just one term. It has essentially the same general features in terms of invariant submanifold and fixed points. The two dimensional fixed point subspace has coordinates

$$
\begin{aligned}
\mathcal{L}= & \left\{\mathbb{G}_{*}, \mathbb{K}_{*}, \Omega_{*}, \mathbb{A}_{*}, \mathbb{B}_{*}\right\} \\
= & \left\{8,0,0, \mathbb{A}_{*}, 1-\frac{192 \alpha \mathbb{A}_{*}^{3}}{576 \mathbb{A}_{*}^{4}+1}\right. \\
& \left.+\gamma\left[\frac{384 \mathbb{A}_{*}^{3}}{576 \mathbb{A}_{*}^{4}+1}-\frac{\ln \left(576 \mathbb{A}_{*}^{4}+1\right)}{3 \mathbb{A}_{*}}\right]\right\},
\end{aligned}
$$

the solution associated to these points is (27).

Using the Hartmann-Grobmann theorem it is easy to prove that, also in this case, the fixed points on the line can 
be either attractors or saddles, with the difference that now the stability depends on $\gamma$ other than $\alpha$. Therefore the same picture of the previous model seems to emerge in this case. The logarithmic correction seems to be irrelevant on Friedmannian dynamics, but will surely be relevant at perturbative level.

\subsubsection{Model 3: $f(\mathcal{G})=\alpha \ln \left[\cosh \left(\chi_{0}^{2} \mathcal{G}\right)\right]-\beta \chi_{0}$}

For the form of $f$ above we have

$$
\begin{aligned}
& \mathbf{X}=\frac{\alpha}{3 \mathbb{A}} \ln \left[\cosh \left(3 \mathbb{A}^{2} \mathbb{G}\right)\right]-\mathbb{B} \\
& \mathbf{Y}=\alpha \mathbb{A} \tanh \left(3 \mathbb{A}^{2} \mathbb{G}\right) \\
& \mathbf{Z}=3 \alpha 2^{6} \mathbb{A}^{3} \operatorname{sech}^{2}\left(3 \mathbb{A}^{2} \mathbb{G}\right) \\
& \mathbf{T}=-2^{9} 3^{2} \alpha \mathbb{A}^{5} \tanh \left(3 \mathbb{A}^{2} \mathbb{G}\right) \operatorname{sech}^{2}\left(3 \mathbb{A}^{2} \mathbb{G}\right)
\end{aligned}
$$

and the dynamical equations read

$$
\begin{aligned}
\frac{\mathrm{d} \mathbb{G}}{\mathrm{d} N}= & \frac{1}{2} \mathbb{G}\left(8-\frac{\mathbb{G}}{\mathbb{K}+1}\right) \\
& +\frac{\mathbb{G} \sinh \left(3 \mathbb{A}^{2} \mathbb{G}\right) \cosh \left(3 \mathbb{A}^{2} \mathbb{G}\right)}{24 \mathbb{A}^{2}(K+9)} \\
& +\frac{\cosh ^{2}\left(3 \mathbb{A}^{2} \mathbb{G}\right)(\mathbb{B}-K+\Omega-1)}{24 \alpha \mathbb{A}^{3}(K+9)} \\
& -\frac{\alpha \cosh ^{2}\left(3 \mathbb{A}^{2} \mathbb{G}\right) \ln \left[\cosh \left(3 \mathbb{A}^{2} \mathbb{G}\right)\right]}{72 \alpha \mathbb{A}^{4}(K+9)}, \\
\frac{\mathrm{d} \Omega}{\mathrm{d} N}= & \Omega\left[\frac{\mathbb{G}}{4(\mathbb{K}+1)}+(3 w+1)\right], \\
\frac{\mathrm{d} \mathbb{K}}{\mathrm{d} N}= & -\frac{\mathbb{K} \mathbb{G}}{4(\mathbb{K}+1)}, \\
\frac{\mathrm{d} \mathbb{A}}{\mathrm{d} N}= & \frac{\mathbb{A}}{4}\left(\frac{\mathbb{G}}{\mathbb{K}+1}-8\right), \\
\frac{\mathrm{d} \mathbb{B}}{\mathrm{d} N}= & -\frac{3 \mathbb{B}}{2}\left(\frac{\mathbb{G}}{\mathbb{K}+1}-8\right) .
\end{aligned}
$$

The system admits again three invariant submanifolds: $\mathbb{K}=$ $0, \Omega=0, \mathbb{A}=0$, and the latter can be singular. These results imply that, in general, no global attractor exists also for this model.

The system (57) presents only a two dimensional fixed point subspace with coordinates

$$
\begin{aligned}
\mathcal{L}= & \left\{\mathbb{G}_{*}, \mathbb{K}_{*}, \Omega_{*}, \mathbb{A}_{*}, \mathbb{B}_{*}\right\} \\
= & \left\{8,0,0, \mathbb{A}_{0}, 1-8 \alpha \mathbb{A}_{*} \tanh \left(24 \mathbb{A}_{*}^{2}\right)\right. \\
& \left.+\frac{\alpha \log \left(\cosh \left(24 \mathbb{A}_{*}^{2}\right)\right)}{3 \mathbb{A}_{*}}\right\},
\end{aligned}
$$

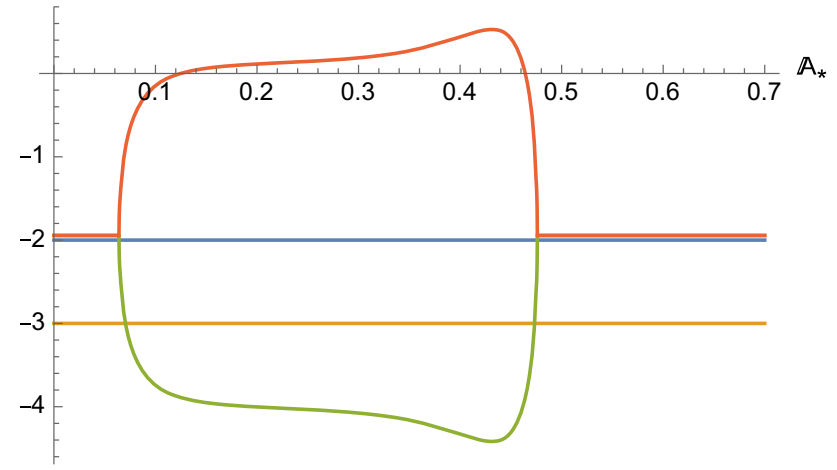

Fig. 5 Plot of the real part of the eigenvalues of the fixed points of the line $\mathcal{L}$ for Model 3. Here $\alpha$ has been chosen to be 3 and $w=0$. Its increase wides the set of values of $\mathbb{A}_{*}$ for which two of the eigenvalues have discordant sign

as in the case of Model 2, the solution associated to this fixed point is (27).

The stability of the fixed points is of the same type to the two previous models, depending essentially on the value of the variable $\alpha$. In Fig. 5 we represent a plot of the eigenvalues of these points.

In spite of the different analytical form, the observed dynamics essentially is the same as in the previous two models. This should not be surprising as all these models have been explicitly designed to have the same characteristics. Our results confirm this fact. As for model 2, differences between the behaviour of these cosmologies will likely become evident at perturbative level.

\section{Conclusions}

In this paper we have proposed a new formalism to analyse the cosmology of Gauss-Bonnet gravity. The method allows us to explore the phase space of any theory, and it associates to the fixed points solutions for the scale factor which present four integration constants.

Many features of this class of theories become clear by looking at the structure of dynamical equations. For example, a transition between decelerating and accelerating cosmology can only happen if the submanifold $\mathbb{G}=0$ is not an invariant submanifold, and therefore the dynamical equations can be used as selection tools for this class of theories, even without the actual phase space analysis. More specifically, we can conclude that all theories for which $\mathcal{G} f^{\prime}(\mathcal{G})-f(\mathcal{G}) \neq 0$ (i.e. $f$ is non-linear) and $f^{\prime \prime}(\mathcal{G})^{-1}=0$ for $\mathcal{G} \rightarrow 0$ will have an invariant submanifold. In other words, the transition between deceleration and acceleration requires a nonlinear function whose second derivative does not diverge in $\mathcal{G}$. This is a general validity criterion of any model of Gauss-Bonnet gravity, and it is fulfilled by the models of Sect. IVB. In fact this condition overlaps with those already found in [25]. Our analysis shows also that there are conditions for which 
another type of fixed point can appear, which could play the role of decelerating fixed point. However, from the first of Eq. (16) we can deduce that the condition for the existence of this point is precisely the same as the one for the existence of the invariant submanifold. This implies that even if this point could be a fixed point associated with a decelerated expansion there would not be heteroclinic orbits connecting it to an accelerated expansion one.

We have applied the new technique to four different models. One of them $f(\mathcal{G})=\alpha \mathcal{G}^{n}$ has been considered for its simplicity and as a testing ground for the new method. The presence of the invariant manifold $\mathbb{G}=0$ forbids the presence of a transition within acceleration and deceleration if $n<2$. Depending on the sign of $n$, there could be two types of attractors: one characterised by the solution (34) $(n<0)$ and the other by (35) $(n>0)$. Since this last solution can lead to a big rip scenario, one can conclude that $n<0$ is a more physically interesting scenario for this model. It is interesting to note that for these values of $n$ the constraints from measurement in the Solar System are known to be much less strict [24].

Next we studied three examples of the models proposed in [25]. They all have very similar phase spaces. This is to be expected from the way in which these models are constructed, and thus gives a confirmation of the consistency of the method we have used. In accordance with the results available in the literature, in this case we find that these models admit a transition from decelerated to accelerated expansion, but that this transition and the approach to the accelerated expansion attractor depend on the initial conditions, and are not guaranteed.

Finally, as in $f(R)$ gravity and because of the higher order of the equations, our analysis shows that there is a set of initial conditions for which these models have a finite time singularity which is an attractor. The presence of this instability also in the Gauss-Bonnet gravity under consideration implies that the existence of this instability is to be ascribed to the fourth-order derivative in the field equations.

\begin{abstract}
Acknowledgements This work was supported by the Fundação para a Ciência e Tecnologia through project IF/00250/2013. SC also acknowledge financial support provided under the European Union's H2020 ERC Consolidator Grant "Matter and strong-field gravity: New frontiers in Einstein's theory" Grant agreement no. MaGRaTh-646597, and under the H2020-MSCA-RISE-2015 Grant No. StronGrHEP-690904. JPM acknowledges the financial support by Fundação para a Ciência e a Tecnologia (FCT) through the research Grant UID/FIS/04434/2013. The authors also acknowledge the COST Action CA15117, supported by COST (European Cooperation in Science and Technology).
\end{abstract}

Open Access This article is distributed under the terms of the Creative Commons Attribution 4.0 International License (http://creativecomm ons.org/licenses/by/4.0/), which permits unrestricted use, distribution, and reproduction in any medium, provided you give appropriate credit to the original author(s) and the source, provide a link to the Creative
Commons license, and indicate if changes were made. Funded by SCOAP ${ }^{3}$.

\section{References}

1. T.P. Sotiriou, V. Faraoni, Rev. Mod. Phys. 82, 451 (2010). arXiv:0805.1726 [gr-qc]

2. S. Capozziello, M. Francaviglia, Gen. Relat. Gravity 40, 357 (2008). arXiv:0706.1146 [astro-ph]

3. S. Nojiri, S.D. Odintsov, Phys. Rep. 505, 59 (2011). arXiv:1011.0544 [gr-qc]

4. S. Capozziello, S. Carloni, A. Troisi, Recent Res. Dev. Astron. Astrophys. 1, 625 (2003). arXiv:astro-ph/0303041

5. V. Faraoni, Cosmology in scalar tensor gravity, (Kluwer Academic Publishers, Dordrecht, The Netherlands, 2004). ISBN-14020-1988-2

6. Y. Fujii, K. Maeda, The scalar-tensor theory of gravitation, (Cambridge University Press, Cambridge, 2003). ISBN-0521811597

7. T. Clifton, P.G. Ferreira, A. Padilla, C. Skordis, Phys. Rep. 513, 1 (2012). doi:10.1016/j.physrep.2012.01.001. arXiv:1106.2476 [astro-ph.CO]

8. P. Avelino et al., Symmetry. 8(8), 70 (2016). doi:10.3390/ sym8080070. arXiv:1607.02979 [astro-ph.CO]

9. Y.F. Cai, S. Capozziello, M. De Laurentis, E.N. Saridakis, Rep. Prog. Phys. 79(10), 106901 (2016). doi:10.1088/0034-4885/79/ 10/106901. arXiv:1511.07586 [gr-qc]

10. G. Calcagni, B. de Carlos, A. De Felice, Nucl. Phys. B 752, 404 (2006). doi:10.1016/j.nuclphysb.2006.06.020. arXiv:hep-th/0604201

11. A. Anabalon, N. Deruelle, D. Tempo, R. Troncoso, Int. J. Mod. Phys. D 20, 639 (2011). doi:10.1142/S0218271811018974. arXiv:1009.3030 [gr-qc]

12. A. Anabalon, N. Deruelle, Y. Morisawa, J. Oliva, M. Sasaki, D. Tempo, R. Troncoso, Class. Quantum Gravity 26, 065002 (2009). doi:10.1088/0264-9381/26/6/065002. arXiv:0812.3194 [hep-th]

13. N. Deruelle, Y. Morisawa, Class. Quantum Gravity 22, 933 (2005). doi:10.1088/0264-9381/22/6/002. arXiv:gr-qc/0411135

14. N. Deruelle, J. Katz, S. Ogushi, Class. Quantum Gravity 21, 1971 (2004). doi:10.1088/0264-9381/21/8/004. arXiv:gr-qc/0310098

15. N. Deruelle, C. Germani, Nuovo Cim. B 118, 977 (2003). doi:10. 1393/ncb/i2004-10015-0. arXiv:gr-qc/0306116

16. N. Deruelle, M. Sasaki, Prog. Theor. Phys. 110, 441 (2003). doi:10. 1143/PTP.110.441. arXiv:gr-qc/0306032

17. N. Deruelle, T. Dolezel, Phys. Rev. D 62, 103502 (2000). doi:10. 1103/PhysRevD.62.103502. arXiv:gr-qc/0004021

18. S. Nojiri, S.D. Odintsov, Phys. Lett. B 631, 1 (2005). doi:10.1016/ j.physletb.2005.10.010. arXiv:hep-th/0508049

19. S. Nojiri, S.D. Odintsov, O.G. Gorbunova, J. Phys. A 39, 6627 (2006). doi:10.1088/0305-4470/39/21/S62. arXiv:hep-th/0510183

20. G. Cognola, E. Elizalde, S. Nojiri, S.D. Odintsov, S. Zerbini, Phys. Rev. D 73, 084007 (2006). doi:10.1103/PhysRevD.73.084007. arXiv:hep-th/0601008

21. S. Nojiri, S.D. Odintsov, P.V. Tretyakov, Phys. Lett. B 651, 224 (2007). doi:10.1016/j.physletb.2007.06.029. arXiv:0704.2520 [hep-th]

22. K. Bamba, A.N. Makarenko, A.N. Myagky, S.D. Odintsov, Phys. Lett. B 732, 349 (2014). doi:10.1016/j.physletb.2014.04.004. arXiv: 1403.3242 [hep-th]

23. N.M. Garcia, T. Harko, F.S.N. Lobo, J.P. Mimoso, Phys. Rev. D 83, 104032 (2011). doi:10.1103/PhysRevD.83.104032. arXiv:1011.4159 [gr-qc]

24. S.C. Davis, AIP Conf. Proc. 957, 379 (2007). doi:10.1063/1. 2823806. arXiv:0708.1967 [hep-th] 
25. A. De Felice, S. Tsujikawa, Phys. Lett. B 675, 1 (2009). doi:10. 1016/j.physletb.2009.03.060. arXiv:0810.5712 [hep-th]

26. A. De Felice, S. Tsujikawa, Phys. Rev. D 80, 063516 (2009). doi:10. 1103/PhysRevD.80.063516. arXiv:0907.1830 [hep-th]

27. A. De Felice, D.F. Mota, S. Tsujikawa, Phys. Rev. D 81, 023532 (2010). doi:10.1103/PhysRevD.81.023532. arXiv:0911.1811 [grqc]

28. P.A.R. Ade et al. [Planck Collaboration], Astron. Astrophys. 594, A13 (2016). doi:10.1051/0004-6361/201525830. arXiv:1502.01589 [astro-ph.CO]

29. See e.g. J.Wainwright, G.F.R. Ellis, Dynamical systems in cosmology (Cambridge University Press, Cambridge, 2005) and references therein. ISBN-0521673526

30. S. Carloni, P.K.S. Dunsby, S. Capozziello, A. Troisi, Class. Quantum Gravity 22, 4839-4868 (2005). arXiv:gr-qc/0410046

31. J.A. Leach, S. Carloni, P.K.S. Dunsby, Class. Quantum Gravity 23 , 4915 (2006). arXiv:gr-qc/0603012

32. S. Carloni, P.K.S. Dunsby, J. Phys. A 40, 6919 (2007). arXiv:gr-qc/0611122

33. M. Abdelwahab, S. Carloni, P.K.S. Dunsby, Class. Quantum Gravity 25, 135002 (2008). arXiv:0706.1375 [gr-qc]

34. S. Carloni, A. Troisi, P.K.S. Dunsby, Gen. Relat. Gravity 41, 1757 (2009). arXiv:0706.0452 [gr-qc]

35. X. Roy, T. Buchert, S. Carloni, N. Obadia, Class. Quantum Gravity 28, 165004 (2011). arXiv:1103.1146 [gr-qc]

36. A. Bonanno, S. Carloni, New J. Phys. 14, 025008 (2012). arXiv:1112.4613 [gr-qc]

37. S. Carloni, S. Vignolo, L. Fabbri, Class. Quantum Gravity 30, 205010 (2013). arXiv:1303.5828 [gr-qc]

38. S. Carloni, S. Vignolo, R. Cianci, Class. Quantum Gravity 31, 185007 (2014). arXiv: 1401.0473 [gr-qc]

39. S. Carloni, E. Elizalde, P.J. Silva, Class. Quantum Gravity 27, 045004 (2010). arXiv:0909.2219 [hep-th]

40. G. Leon, E.N. Saridakis, JCAP 1504(04), 031 (2015). arXiv: 1501.00488 [gr-qc]
41. M.A. Skugoreva, E.N. Saridakis, A.V. Toporensky, Phys. Rev. D 91(4), 044023 (2015). arXiv:1412.1502 [gr-qc]

42. G. Kofinas, G. Leon, E.N. Saridakis, Class. Quantum Gravity 31, 175011 (2014). arXiv:1404.7100 [gr-qc]

43. C.R. Fadragas, G. Leon, E.N. Saridakis, Class. Quantum Gravity 31, 075018 (2014). arXiv:1308.1658 [gr-qc]

44. G. Leon, E.N. Saridakis, JCAP 1303, 025 (2013). arXiv:1211.3088 [astro-ph.CO]

45. G. Leon, E.N. Saridakis, Class. Quantum Gravity 28, 065008 (2011). arXiv:1007.3956 [gr-qc]

46. P. Fiziev, D. Georgieva, Phys. Rev. D 67, 064016 (2003)

47. L. Amendola, R. Gannouji, D. Polarski et al., Phys. Rev. D 75, 083504 (2007). arXiv:gr-qc/0612180

48. S. Carloni, A. Troisi, P.K.S. Dunsby, Gen. Relat. Gravity 41, $1757-$ 1776 (2009). arXiv:0706.0452 [gr-qc]

49. S. Carloni, T. Koivisto, F.S.N. Lobo, Phys. Rev. D 92(6), 064035 (2015). doi:10.1103/PhysRevD.92.064035. arXiv:1507.04306 [grqc]

50. S. Carloni, F.S.N. Lobo, G. Otalora, E.N. Saridakis, Phys. Rev. D 93, 024034 (2016). doi:10.1103/PhysRevD.93.024034. arXiv:1512.06996 [gr-qc]

51. A. Alho, S. Carloni, C. Uggla, JCAP 1608(08), 064 (2016). doi:10. 1088/1475-7516/2016/08/064. arXiv:1607.05715 [gr-qc]

52. J.D. Barrow, S. Hervik, Phys. Rev. D 74, 124017 (2006). doi:10. 1103/PhysRevD.74.124017. arXiv:gr-qc/0610013

53. S. Carloni, JCAP 1509(09), 013 (2015). doi:10.1088/1475-7516/ 2015/09/013. arXiv:1505.06015 [gr-qc]

54. J. Guckenheimer, P. Holmes, Nonlinear Oscillations, Dynamical Systems, and Bifurcations of Vector Fields, Applied Mathematical Sciences (Springer, New York, 1982), pp. 13 and 18

55. S.H. Strogatz, Nonlinear Dynamics and Chaos: With Applications to Physics, Biology, Chemistry, and Engineering (Perseus Books, Reading, 1994), p. 155 